Available online on 15.03.2017 at http://iddtonline.info
¿ 2011-17, publisher and licensee JDDT, This is an Open Access article which permits unrestricted
noncommercial use, provided the original work is properly cited

Review Article

\title{
WOMEN SPECIFIC VARIABLES IN CARDIOVASCULAR DISEASES
}

\author{
Zalak S Dodiya ${ }^{1}$, Dr Rina N Desai ${ }^{2}$
}

Shri Sarvajanik Pharmacy College, Mehsana, Gujrat, India

\begin{abstract}
Over the past decade or more, the prevalence of traditional risk factors for cardiovascular diseases has been increasing in the major populous countries of the developing world, including India, with consequent increases in the rates of coronary and cerebrovascular events. Cardiovascular diseases are predicted to be the major causes of morbidity and mortality in most developing nations around the world. Historically, most populations from South Asia have documented low levels of CV risk factors, particularly blood lipid levels, diabetes, and hypertension. However, in recent decades, the prevalence of these risk factors has increased, especially in urban areas. Numerous differences exist between younger and older women and between women and men with respect to the pathology of $\mathrm{CHD}$ and its incidence and prevalence over the life cycle. Women with obesity, metabolic syndrome, or diabetes have lipid profiles that adversely affect CHD risk.
\end{abstract}

Key words: cardiovascular disease, women specific variables.

\section{INTRODUCTION:}

Despite an overall reduction in the death rate due to cardiovascular disease (CVD) in the United States over the last several decades, the rate of decline is less for women than men and less for African-American women than white women. ${ }^{1}$ Due to an aging population, the absolute number of deaths due to CVD in women is actually increasing. Because the risk of CVD increases with age, there is a need for an increased awareness of the importance of CVD as a major public health issue for older women.

CVD, particularly coronary heart disease (CHD) and stroke, remains the leading killer of women in most developed countries. CVD is a particularly important problem among minority women. This article discusses recent advances in knowledge of the occurrence, determinants, and treatment of atherosclerotic CVD in women, including CHD, hypertension, stroke, and peripheral arterial disease and much attention has been directed toward a better appreciation of the influence of gender on cardiovascular risk and management, but important gaps in knowledge remain. Recent developments in cardiovascular research undoubtedly will have a significant impact on prevention, clinical care, and outcomes of women and will provide direction for future work. ${ }^{2}$

\section{Secondary Prevalence of CVD:}

Secondary prevention of CVD refers to healthcare that aims to prevent the recurrence of cardiovascular events (e.g. heart attack or stroke) or complications of CVD in people diagnosed with $\mathrm{CVD}^{3}$.

Secondary prevention strategies for CVD are modification of behavioral risk factors, psychological care, and adherence to prescribed medicines, education and support for self-management, medical care, cardiac rehabilitation services, and multidisciplinary $\mathrm{CHF}$ management services

The prevalence of different "lifestyle" and biological CVD risk factors across the country - and results show that these risk factors are now at higher levels in India 
than in developed countries and regions such as the USA and Western Europe ${ }^{4}$.

The prevalence of coronary heart disease (CHD) is known to be very high among Indians, both in India and abroad. Moreover, among Indians, CHD occurs at least a decade or 2 earlier compared with Europeans. The reason for the increased susceptibility of Indians to CHD is yet to be completely understood. However, several studies have hinted that the clustering of risk variables (mechanism of which is still unknown) of metabolic syndrome (MS) could be responsible for the increasing incidence of CHD among Indians. This includes central obesity, hypertriglyceridemia, less levels of high-density lipoprotein cholesterol, high blood pressure, and high levels of fasting blood glucose.

Secondary prevention relies on early detection of disease process and application of interventions to prevent progression of disease. ${ }^{4}$

There is a significantly lower age-specific risk of CHD in women than men. Risk of death due to CHD in women is roughly similar to that of men 10 years younger. Despite their marked advantage in age-specific risk of CHD death, the greater likelihood of survival of women to advanced ages produces nearly equal numbers of actual deaths due to CHD in men and women.

Treatment efforts in women may be less likely to be effective in reducing the death rate because the majority of cases may never reach a hospital. For these women, primary prevention is likely to be the only practical solution. Prevalence of ECG-defined MI shows similar trends with comparable rates among women in the two ethnic groups but higher rates in non-Hispanic white men compared with Hispanic men. More recent data from the Corpus Christi Heart Project demonstrate higher hospitalization rates for heart attacks and incident MI in Mexican-Americans compared with non-Hispanic whites for both men and women. This same study demonstrated a higher case-fatality after MI in MexicanAmericans and women compared with non-Hispanic whites and men, respectively.

\section{WOMEN SPECIFIC VARIABLES:}

\section{Preeclampsia:}

The association between preeclampsia and cardiovascular disease has been an increasing area of interest over the last years. Cardiovascular disease is the number one cause of death in women in the western world and more women than men die of heart disease each year. The most common pregnancy disorder is preeclampsia. Preeclampsia is defined by hypertension and de novo proteinuria and remains responsible for high maternal and fetal morbidity and mortality worldwide. Pregnancy has been described as a "stress test" for future cardiovascular disease, to identify women young enough to benefit from screening. Women with a history of early onset (severe) preeclampsia have the highest risk of cardiovascular disease later. However, the exact underlying link between the two disorders is still unknown. In this review we describe different facets of the association between preeclampsia and cardiovascular disease and we give an overview of the recent literature ${ }^{4}$.

\section{Gestational diabetes:}

Gestational diabetes, which develops only during pregnancy and usually disappears after the pregnancy, increases the risk that the mother will develop diabetes later. The condition is managed with meal planning, activity and sometimes insulin or other medications.

Pregnant women who develop gestational diabetes may be more at risk of developing heart disease later in life, according to new research ${ }^{5}$.

Gestational diabetes only occurs during pregnancy. It happens when hormones activated during pregnancy weaken the effect of insulin, the hormone that normally allows cells to absorb glucose from the blood.

Factors that can make pregnant women at greater risk of getting gestational diabetes include having a family history of diabetes, having previously had an unexplained miscarriage, being older than 25 when they became pregnant or being overweight before becoming pregnant.

Women who develop gestational diabetes are usually able to control their blood sugar without harming their baby's health. But having gestational diabetes does make women more likely to develop diabetes 5-10 years after giving birth.

The new study finds that women who get gestational diabetes while pregnant also have an associated risk of atherosclerosis - where the arteries around the heart become clogged by fatty substances. Because atherosclerosis disrupts the flow of blood to and from the heart, this can eventually cause heart attacks and other cardiovascular diseases ${ }^{6}$.

\section{Autoimmune disease:}

Autoimmune diseases (AIDs) have been associated with accelerated atherosclerosis (AT) leading to increased cardio- and cerebrovascular disease risk. Traditional risk factors, as well as systemic inflammation mediators, including cytokines, chemokines, proteases, autoantibodies, adhesion receptors, and others, have been implicated in the development of these vascular pathologies. Yet, the characteristics of vasculopathies may significantly differ depending on the underlying disease. In recent years, many new genes and signalling pathways involved in autoimmunity with often overlapping patterns between different disease entities have been further detected. Epigenetics, the control of gene packaging and expression independent of alterations in the DNA sequence, is providing new directions linking genetics and environmental factors. Epigenetic regulatory mechanisms comprise DNA methylation, histone modifications, and microRNA activity, all of which act upon gene and protein expression levels. Recent findings have contributed to our understanding of how epigenetic modifications could influence AID development, not only showing differences between AID patients and healthy controls, but also showing how one disease differs from another 
and even how the expression of key proteins involved in the development of each disease is regulated ${ }^{7}$.

\section{Thyroid diseases:}

Common cardiovascular symptoms associated with thyroid conditions include a rapid heart rate, angina pectoris, atrial fibrillation, and congestive heart failure. Cardiac symptoms can be seen in anybody with hypothyroidism but are especially likely in an individual who already has underlying heart disease (Figure). Subclinical hyperthyroidism is associated with important cardiovascular risk factors, yet there are conflicting results on cardiovascular mortality ${ }^{8}$.

\section{Rheumatoid arthritis:}

The increased mortality in rheumatoid arthritis (RA) is mainly due to (atherosclerotic) cardiovascular disease. The cardiovascular morbidity is also increased in comparison with the general population. This increased cardiovascular burden could be caused by 1) an enhanced prevalence of cardiovascular risk factors 2) under treatment of cardiovascular risk factors or 3) RA itself, particularly due to its chronic inflammatory component. Cardiovascular risk factors only partially explain the increased cardiovascular risk and it is becoming increasingly acknowledged that the underlying inflammation in RA plays an essential role. This is probably related to the fact that atherosclerosis also has an inflammatory etiology that is accelerated by RA. Similarly, it can be expected that effective suppression of this inflammatory process by disease modifying antirheumatic drugs and/or biologicals lowers the cardiovascular risk. Altogether, there is accumulating evidence that the increased cardiovascular risk in RA is comparable to that of type 2 diabetes and actually RA should be seen as a new, independent, cardiovascular risk factor for which cardiovascular risk management is essential ${ }^{9}$.

\section{Gout:}

Gout is known to boost the risk of a heart attack in men. But to date, little has been known about the impact of gout on women's cardiovascular health.

Gout is common and caused by inflammation in the joints as a result of excess uric acid deposits. Uric acid is a by-product of purines, which are abundant in a Western diet.

Obesity, weight gain, high alcohol intake, high blood pressure, poorly functioning kidneys and certain drugs can all precipitate its development.

Compared with women who did not have gout, those who did were $39 \%$ more likely to have a heart attack of any kind and $41 \%$ more likely to have a non-fatal heart attack.

The risks were significantly higher among the women than among the men. Men with gout were only $11 \%$ more likely than those without the disease to have a fatal or non-fatal heart attack.

The findings held true after adjusting for factors likely to influence the results, such as age, other underlying health problems, and use of prescription drugs ${ }^{10}$.
The authors comment that excess uric acid may boost levels of inflammation and platelet stickiness, both of which are implicated in coronary artery heart disease. Other forms of arthritis also boost the risk of cardiovascular disease ${ }^{10}$.

\section{Hormone therapy:}

The net benefit of estrogen or combined estrogenprogestin replacement therapy in postmenopausal women is still uncertain. Based upon extensive observational data, it was believed that estrogen was cardioprotective; as a result, estrogen therapy was routinely prescribed for both primary and secondary prevention of coronary heart disease (CHD) ${ }^{11}$.

Normal women have menopause at a mean age of 51 years, with 95 percent becoming menopausal between the ages of 45 to 55 years. Estrogen is the most effective treatment available for relief of menopausal symptoms, most importantly, hot flashes. Postmenopausal hormone therapy (estrogen alone or combined with a progestin) is currently indicated for management of menopausal symptoms $^{12}$.

\section{Psoriasis:}

There is considerable evidence to show that patients with moderate-to-severe psoriasis have a significantly increased risk of cardiovascular disease and cardiovascular risk factors such as obesity, diabetes mellitus, the metabolic syndrome and smoking compared to the general population. The mechanistic link between psoriasis and this observed increase in cardiovascular co-morbidities has not been fully defined. It is clear, however, that common inflammatory pathways are at play in the pathophysiology of psoriasis, obesity and coronary artery disease. It had been proposed that the control of systemic inflammation in psoriasis could help reduce cardiovascular morbidity, and retrospective studies of methotrexate and anti-TNFa agents have suggested a cardio-protective effect with use of these agents. More recently, however, there have been concerns regarding a potential excess of cardiovascular events with the newer generation of antiinterleukin-12p40 antibodies. In this article we review the association of psoriasis with cardiovascular disorders and the effects of current psoriasis therapies on cardiovascular risk ${ }^{13}$.

\section{Pregnancy induced hypertension:}

Women with a history of pregnancy-induced hypertension (including preeclampsia) are at increased risk of cardiovascular and metabolic diseases later in life. However, it is not known whether the increased risk can be attributed to factors that originate in pregnancy or to prepregnancy factors that are associated with both the risk of preeclampsia and gestational hypertension and the subsequent risk of cardiovascular and metabolic diseases $^{14}$.

\section{Risk Factors and Primary Prevention}

The major risk factors for CHD in women are cigarette smoking, hypertension (including isolated systolic hypertension), dyslipidemia, diabetes mellitus, obesity, sedentary lifestyle, and poor nutrition. Although most 
risk factors for CHD are similar in men and women, gender differences have been documented, particularly diabetes and dyslipidemia. The prevalence, magnitude of effect, and gender differences in identified risk factors for CHD are summarized below.

Cigarette smoking remains the leading preventable cause of CHD in women, with more than $50 \%$ of MIs among middle-aged women attributable to tobacco. ${ }^{16}$ Risk of CHD begins to decline within months of smoking cessation and reaches the level of persons who have never smoked within 3 to 5 years. Smoking cessation rates have declined more slowly among women than men. On the basis of current trends, it is estimated that smoking rates will be higher in women than in men. These changing demographics of smoking, particularly the unfavorable smoking patterns among younger women, may contribute substantially to the future burden of CHD on women, as well as other smoking-related illnesses.

Epidemiological studies document a strong association between high levels of both systolic and diastolic blood pressure (BP) and risk of CHD in both women and men. Among adults older than 45 years, having hypertension (defined as either taking antihypertensive medication or having systolic $\mathrm{BP} \geq 140 \mathrm{~mm} \mathrm{Hg}$ or diastolic $\mathrm{BP}>90 \mathrm{~mm}$ $\mathrm{Hg}$ ). approximately one half of identified hypertensive subjects were receiving treatment with antihypertensive medication, but only about some had hypertension that was well controlled (BP <140/90). Of particular concern for older women is isolated systolic hypertension, which is estimated to affect some of women older than 65 . For severe diastolic hypertension (diastolic $\mathrm{BP} \geq 110$ ), the benefits of medication are large and obvious for both sexes. Primarily $\beta$-blockers and thiazide diuretics were used, shows a significant effect for stroke and major CVD events in women. The relative risk reduction did not differ between men and women. Weight reduction and dietary interventions also have important roles in prevention and treatment of hypertension. ${ }^{17}$

Increased total serum cholesterol and low-density lipoprotein (LDL) cholesterol are risk factors for CHD in both women and men. From 1980 to 1991 more than $50 \%$ of women older than 55 years had serum cholesterol levels that were considered high $(>240$ $\mathrm{mg} / \mathrm{dL}$ ). A low level of high-density lipoprotein (HDL) cholesterol, however, was a risk factor for CHD in both younger and older women and was a stronger predictor of CHD mortality in women than in men. The role of triglycerides in CHD risk remains controversial, but observational studies suggest they may be a particularly important risk factor in women and the elderly, the majority of whom are women. ${ }^{18}$

Obesity and sedentary lifestyle are parallel, interrelated epidemics that contribute to increased risk of CHD. The prevalence of obesity has increased among both men and women in the past decade; currently about one third of adult women are classified as obese. Moreover, $60 \%$ of both men and women have no regular physical activity. Obesity, particularly abdominal adiposity, is an important risk factor for CHD in women. Although most of the epidemiological studies of exercise and CHD have been conducted in men, most studies in women suggest a comparable $50 \%$ risk reduction among active women compared with sedentary women. Recent evidence suggests that even moderate-intensity activity, including brisk walking, is associated with substantial reduction in CHD risk. ${ }^{19}$

Regular exercise and maintenance of healthy weight should also help reduce insulin resistance and the risk of non-insulin-dependent diabetes mellitus, which appears to be an even stronger risk factor for CHD in women than in men. Diabetes is associated with a threefold to sevenfold elevation in CHD risk among women, compared with a twofold to threefold elevation among men; this gender-based difference may be due to a particularly deleterious effect of diabetes on lipids and blood pressure in women. Diabetes is the fourth leading cause of death among black women and third among Hispanic women aged 45 to 74 years and AmericanIndian women aged 65 to 74 years. It is also the second leading cause of death in Pima Indian women. Approximately half of all deaths in persons with noninsulin dependent diabetes mellitus are due to heart disease, the majority of which is ischemic heart disease. $^{20}$

Increasing research and knowledge related to nutrition have led to identification of several dietary factors that influence CHD risk. The epidemiological evidence is compelling: diets low in saturated fat and high in fruits, vegetables, whole grains, and fiber are associated with a reduced risk of CHD. Trans fatty acids have recently been linked to adverse lipid profiles and an increased risk of CHD. The role of other fatty acids, including monounsaturated, polyunsaturated, and marine omega-3 fatty acids, remains controversial. Moderate intake of alcohol is related to reduction of $\mathrm{CHD}$ but may raise blood pressure and increase risk of breast cancer. ${ }^{21}$

Genetic factors are also important determinants of CHD risk in both men and women, but they are not modifiable and thus are not further addressed here. Recently identified thrombotic, hemostatic, and inflammatory markers for CHD have promising roles in predicting risk of vascular events, but their clinical usefulness in the general population remains largely unknown.

Pharmacological interventions, including antiplatelet therapy and postmenopausal hormone replacement therapy, are appropriate in selected patients. In primary prevention the balance of benefits and risks of aspirin prophylaxis among women remains unknown and awaits the results of the ongoing Women's Health Study, the only randomized trial of aspirin in usual-risk women. Antioxidant vitamin supplements, particularly vitamin $\mathrm{E}$ and homocysteine-lowering agents such as folate and $\mathrm{B} 6$, have promising roles in prevention of CHD, but conclusive evidence awaits the results of several ongoing randomized clinical trials. $\frac{32}{\text { Early }}$ surgical menopause is linked to increased risk of CHD, which appears to be negated by the use of estrogen therapy. ${ }^{22}$

\section{Cardiac Rehabilitation}

A recent secondary prevention consensus panel of the AHA found compelling scientific evidence that 
comprehensive risk factor interventions in patients with CHD extend overall survival, improve quality of life, decrease the need for interventional procedures, and reduce the incidence of subsequent MI. Cardiac rehabilitation is a key step initiating the process of risk reduction and restoration of functional capacity after a heart attack or revascularization. It is an established treatment that includes exercise training, risk factor modification, and psychosocial and vocational counseling. Studies that have included women suggest they have similar improvements in functional capacity and other outcomes compared with men. ${ }^{23}$

\section{Stroke}

\section{Epidemiology}

Stroke represents a significant healthcare burden for our society. It is the third leading cause of death in the United States and the leading cause of disability, with an estimated direct cost of $\$ 41$ billion per year. Healthcare costs increase dramatically as stroke severity increases, with greatest costs occurring for those patients with subarachnoid hemorrhages, the only subtype of stroke more common in women than in men.Strokes account for half of acute neurological hospitalizations. Stroke survivors are at greater risk of dementia and disability than men and women without a history of stroke. ${ }^{24}$

The overall prevalence of stroke is higher in men than in women but increases with age in both gender groups. However, the incidence of ischemic stroke for persons with a history of transient ischemic attacks (TIA) is higher in women than in men. Seasonal effects on the incidence of stroke in men and women have been suggested but not confirmed by all studied. ${ }^{25}$

Although the lifetime risk of stroke is higher in men, women are more likely to die of stroke, probably due to their older age at its occurrence and their longer life expectancy. Over an entire lifetime, about $16 \%$ of women will die of stroke, whereas only $8 \%$ of men will die of stroke. However, mortality rates over the last decades have been declining for both men and women ${ }^{25}$.

\section{Risk Factors}

Many identifiable risk factors for ischemic stroke in both men and women have been fairly consistent over epidemiological studies. They include hypertension, smoking, diabetes mellitus, ischemic heart disease, atrial fibrillation, and TIAs. Most epidemiological studies have not provided gender-specific relative risks

Hypertension is clearly a major risk factor for stroke, with a $46 \%$ increase in stroke risk for every $7.5 \mathrm{~mm} \mathrm{Hg}$ increase in diastolic blood pressure. In black women the risk of developing hypertension is more than twice that of white women. Current smoking habits are related to stroke risk for both men and women even after adjustment for age, gender, and hypertension ${ }^{26}$.

Diabetes mellitus is associated with a doubling of ischemic stroke risk. Not only does diabetes increase stroke risk, it increases severity and mortality associated with stroke ${ }^{27}$.

\section{Treatment}

To date there are no specific recommendations for treatment of stroke in women, and recommendations for acute interventions for women and men appear to be the same. For all stroke victims, early detection and treatment are vital. Thrombolytic therapy with r-TPA for acute ischemic stroke can be effective for ischemic stroke patients when administered early in the course of the event. Recently, a panel of the American Heart Association Stroke Council reviewed the clinical trials of thrombolytic drugs and established guidelines for management of patients with acute ischemic stroke. The guidelines call for early administration of r-TPA when the time of stroke onset can be ascertained reliably and with some specific exclusions. Ten percent of patients with acute stroke develop hemorrhage with r-TPA. The panel also considered the need for careful attention to ancillary care measures and management of bleeding complications, and these are outlined in the guidelines. Although treatment after the acute stage should focus on the patient's particular risk factor profile and the pathogenesis of the stroke, most care will include the use of antiplatelet aggregating or antithrombotic agents such as aspirin, ticlopidine, or warfarin. Secondary prevention may include aspirin for both men and women. While women and men both received equal benefit with aspirin therapy in this trial of secondary prevention, the efficacy of aspirin use for primary stroke prevention in women remains questionable. In patients with TIA and atrial fibrillation, adjusted dose warfarin may prevent stroke. ${ }^{28}$

\section{Peripheral Artery Disease \\ Epidemiology}

Peripheral artery disease is more prevalent in women than generally appreciated, but estimates vary greatly according to the diagnostic criteria applied. Prevalence and incidence rates do not differ significantly by gender, although incidence rates in women lag behind those in men in a pattern similar to that for coronary disease. The incidence of peripheral artery disease increases sharply with age regardless of the criteria applied. In the Framingham study, age-specific incidence accelerated rapidly in women. Intermittent claudication is not a very sensitive indicator of disease; when used as the sole criterion, it yields prevalence rates of just $2 \%$ to $3 \%$. Absence of pulses is also not a sensitive indicator. ${ }^{29}$ Data from a variety of studies clearly show that the risk of cardiovascular events associated with decrements in peripheral vascular function is continuous.

Besides claudication, commonly used criteria for diagnosing peripheral arterial disease include an ankle/brachial index $(\mathrm{ABI}) ;<0.9$ determined by the ratio of systolic BP at the posterior tibial artery at the level of the ankle divided by the brachial artery pressure; flow velocity deficits measured by Doppler; the absence of posterior tibial or dorsalis pedis pulses; and intermittent claudication. In studies in which at least two of these criteria were used, prevalence rates ranged from $6 \%$ to $20 \%$. Asymptomatic patients have survival rates that are not significantly different from those with symptoms, suggesting that claudication is not a reliable marker for 
severity of disease. Thus, when peripheral artery disease is considered a problem only in the presence of claudication, the majority of the population at risk is discounted. The ABI and measurements with a handheld Doppler in an outpatient setting can reliably diagnose the majority of peripheral artery disease. ${ }^{30}$

\section{Risk Factors}

Smoking is the most consistently associated risk factor, and prevalence rates in nonsmokers are less than half that of smokers. Other risk factors include glucose intolerance, increased body mass index, elevated blood pressure, and elevated fibrinogen. The association between peripheral artery disease and lipids is less definitive. Except for the importance of smoking, the risk factor profile common in peripheral artery disease shares features with the metabolic syndrome, glucose intolerance with low HDL cholesterol and often normal levels of LDL cholesterol, with a large fraction of the LDL cholesterol in a dense, more atherogenic form. An association with the metabolic syndrome in persons whose total and LDL cholesterol levels are normal may explain the inconsistent associations for lipids.

The degree of overlap in risk factors between peripheral artery disease and other major atherosclerotic CVD varies considerably by population and the diagnostic criteria applied. In the Cardiovascular Health Study peripheral artery disease was associated with increased carotid wall thickness, abnormal cardiac wall motion, and major electrocardiographic abnormalities. The Atherosclerosis Risk in Communities Study found peripheral artery disease to be the most important predictor of carotid thickness. Similarly, $25 \%$ of women with a history of coronary disease or stroke had peripheral artery disease, compared with an $11 \%$ prevalence of peripheral artery disease in women without this history. Typically, the more detailed the assessment of the arterial tree, the greater the concordance between peripheral arterial, coronary, and cerebrovascular diseases.

Peripheral artery disease itself is a strong risk factor for other cardiovascular events and mortality. There is a threefold to fourfold increase in relative risk for allcause mortality associated with peripheral arterial disease that does not differ by gender. The relative risk for cardiovascular death is between 4 and 6 , and the relative risk for CHD death is between 4 and 7 . Moreover, adjustment for risk factors and exclusion of persons with a history of other CVD at baseline does not attenuate these risks, suggesting that once manifested, the disease itself is an independent predictor of survival. $^{31}$

\section{Treatment}

Smoking cessation is essential for any patient still smoking when peripheral artery disease is recognized. Antiplatelet therapy may be effective in reducing adverse outcomes. Surgery is indicated for severe disease and may reduce direct sequelae of poor peripheral circulation, but, just as for coronary disease, a comprehensive program of atherosclerotic risk reduction and exercise is necessary to reduce risk of other cardiovascular events. Despite ambiguity about the association between lipids and peripheral artery disease, lipid-lowering therapy is likely to be protective. ${ }^{32}$

\section{Women and Coronary Artery Disease}

In the United States, CHD is the leading cause of death in both men and women, claiming more lives than cancer, accidents, and diabetes combined. Although breast cancer may be more feared, age-adjusted death rates from CVD in women are 4 times higher in white women and 6 times higher in black women than the death rates for breast cancer.

The 2010 ACCF/AHA report on assessment of cardiovascular risk in asymptomatic adults includes the recommendation that for all adult women and men, global risk scoring should be performed and a family history of cardiovascular disease should be obtained for cardiovascular risk assessment. $^{33}$

Compared with men, LDL cholesterol is lower and HDL cholesterol is higher in women before menopause. Although women have lower rates of hypertension and cigarette smoking than men, rates for obesity and diabetes mellitus are higher. Diabetes mellitus is a particularly serious risk factor in women, tripling the risk of cardiovascular death and causing diabetic women to have the same frequency of CVD as diabetic men. HDL cholesterol and triglyceride levels are more predictive of CVD in women than in men. Women have been noted to have similar or slightly higher prevalence of stable angina as compared to men.

It is now known that women tend to present more commonly with unstable angina as compared to men, the reverse of which is true for MI. However, when women do present with MI, they are more likely to have $\mathrm{Q}$ wave rather than non-Q wave.-Mortality rates of MI and $\mathrm{CABG}$ are about $50 \%$ higher in women, mostly related to older age of onset. Lipid lowering has shown similar efficacy in women and men in the angiographic progression and event trials. Cardio protective agents, including aspirin, beta-blockers, and ACE inhibitors, appear to have similar efficacy in men and women.

Hormone therapy is no longer recommended to prevent coronary events in postmenopausal women with or without established CHD. Although hormone therapy improves LDL and HDL cholesterol levels, it also increases coagulation and inflammation (as measured by C-reactive protein [CRP]) and decreases LDL particle size. Treatment rates for risk factors in women tend to be even lower than in men, as are rates for coronary angiography and coronary artery revascularization following presentation with chest pain.

Women who may have had radiotherapy through the mid-1980s to treat breast cancer are also at an increased risk of mortality from cardiovascular disease. The concern is even greater if the woman was treated for a left-sided breast cancer with contemporary tangential breast or chest wall radiotherapy.

Finally, it must be emphasized that while the guidelines detailed above represent best practice, their formulation is often a blend of science and art. Therefore, guideline 
interpretation should always occur alongside good clinical judgment ${ }^{34}$.

\section{DISCUSSION}

CVD in women will continue to be a public health priority as significant numbers of aging women are at increased risk for morbidity and mortality related to CVD. Healthcare systems need to begin to shift paradigms to emphasize healthy lifestyles for young women. This approach will help prevent development of risk factors and minimize the need to manage them at a later time. Healthcare providers need to be sensitive to gender differences in presentation, prognosis, and responsiveness to treatment of CVD. Scientists are encouraged to continue to examine potential differences between men and women in the pathophysiology and clinical outcomes of CVD. More research in minority women is of particular importance, given the high level of risk factors and mortality rates in this population. Health educators will play a pivotal role in communicating and translating scientific developments about women and heart disease. Public policy makers should take the lead in ensuring that women of diverse backgrounds and circumstance have equal access to

\section{REFERENCES:}

1. National Heart Foundation of Australia. Secondary prevention of cardiovascular disease: a call for action to improve the health of Australians. National Heart Foundation of Australia, Melbourne; 2010.

2. Hermes W, Van Kesteren F, De Groot CJ, Preeclampsia and cardiovascular risk,Minerva Ginecol, 2012 Aug; 64(4):28192.

3. Gestational diabetes may raise risk for heart disease in midlife, American Heart Association Rapid Access Journal Report, March 12, 2014.

4. David McNamee, Gestational diabetes may increase heart disease risks for pregnant women, J Am Heart Assoc, doi: 10.1161/JAHA.113.000490, March 2014.

5. Chary López-Pedrera, Carlos Pérez-Sánchez, Manuel RamosCasals, Monica Santos-Gonzalez, Antonio Rodriguez-Ariza et al Cardiovascular Risk in Systemic Autoimmune Diseases: Epigenetic Mechanisms of Immune Regulatory Functions, Clinical and Developmental Immunology, Volume 2012 (2012), Article ID 974648, 10 pages.

6. http://www.physiciansweekly.com/thyroid-disorderscardiovascular-risks/\#sthash.RnHUJvSW.dpuf

7. Nurmohamed MT, Cardiovascular risk in rheumatoid arthritis, Autoimmun Rev. 2009 Jul; 8(8):663-7. doi: 10.1016/j.autrev.2009.02.015. Epub 2009 Feb 12.

8. Women with gout at greater risk of heart attack than men, BMJ-British Medical Journal, February 11, 2010.

9. Kathryn A Martin, Robert S Rosenson, Postmenopausal hormone therapy and cardiovascular risk, UpToDate, feb 2014.

10. Kathryn A Martin, Robert L Barbieri, Postmenopausal hormone therapy: Benefits and risks, UpToDate, feb 2014.

11. Ryan C, Menter A., Psoriasis and cardiovascular disorders, G Ital Dermatol Venereol, pubmed, 2012 Apr; 147(2):179-87.

12. Pål R. Romundstad, Elisabeth B. Magnussen, George Davey Smith and Lars J. Vatten, Hypertension in Pregnancy and Later Cardiovascular Risk: Common Antecedents?, (C) 2010 American Heart Association,Circulation, 2010; 122:579-584.

13. Mosca L, Benjamin E J, Berra K, Bezanson J L, Dolor R J, Reasons for India's growing cardiovascular disease epidemic pinpointed in largest-ever risk factor study, world heart federation, April, 2012.

14. Goff DC, Nichaman MZ, Chan W, Ramsey DJ, Labarthe DR, Ortiz C. Greater incidence of hospitalized myocardial care. It is only through a multifaceted approach that cardiovascular science and medicine can be advanced for the betterment of all.

\section{CONCLUSION}

Evidence suggests that CVD can be prevented in women. Clinical recommendations are provided to assist healthcare providers and the public in efforts to avoid an initial or recurrent cardiovascular event. Our systematic search of the literature shows that several prevention strategies are likely to have substantially greater benefit than risk and that some interventions are likely to be associated with greater risk than benefit. It is important that the public be appropriately informed about potentially lifesaving preventive therapies and take action to lower their risk. On the basis of our review of the scientific evidence, it appears the risk of no action is far greater than that of applying knowledge to prevent CVD. Moreover, elderly women, especially those over 80 , in whom CVD is common. These results suggest that identification of hypertension in women should dictate additional CAD risk assessment and risk factor management. infarction among Mexican Americans than non-Hispanic whites. The Corpus Christi Heart Project. 1988-1992. Circulation. 1997; 95:1433-1440.

15. Willett WC, Green A, Stampfer MJ, Speizer FE, Colditz GA, Rosner B, Monson RR, Stason W, Hennekens CH. Relative and absolute excess risks of coronary heart disease among women who smoke cigarettes. N Engl J Med. 1987; 317:1303-1309.

16. Whelton PK, He J, Appel LJ. Treatment and prevention of hypertension. In: Manson JE, Ridker PM, Gaziano JM, Hennekens $\mathrm{CH}$, eds. Prevention of Myocardial Infarction. New York, NY: Oxford University Press, 1996:154-171.

17. LaRosa JC. Triglycerides and coronary risk in women and the elderly. Arch Intern Med. 1997; 157:961-968.

18. Manson JE, Lee I-M. Exercise for women: how much pain for optimal gain? N Engl J Med. 1996; 334:1325-1327.

19. Geiss LS, Herman WH, Smith PJ. Mortality in non-insulindependent diabetes. In: Diabetes in America. National Institutes of Health, National Institute of Diabetes and Digestive and Kidney Disease; 1995:249-250.

20. Fuchs FC, Stampfer MJ, Colditz GA, Giozannucci EL, Manson JE, Kawachi I, Hunter DJ, Hankinson PE, Hennekens CH, Rosner B, Speizer FE, Willett WC. A prospective study of alcohol consumption and mortality among women. N Engl J Med. 1995:332; 1245-1250.

21. Colditz GA, Willett WC, Stampfer MJ, Rosner B, Speizer $\mathrm{FE}$, Hennekens $\mathrm{CH}$. Menopause and the risk of coronary heart disease in women. N Engl J Med. 1987; 316:11051110.

22. Cannistra LB, Balady GJ, O’Malley CJ, Weiner DA, Ryan TJ. Comparison of the clinical profile and outcome of women and men in cardiac rehabilitation. Am J Cardiol. 1992; 69:1274-1279.

23. Prencipe M, Ferretti C, Casini AR, Santini M, Giubilei F, Culasso F. Stroke, disability, and dementia. Results of a population survey. Stroke. 1997; 28:531-536.

24. Van Weel C, van de Lisdonk E, van den Bosch W, van den Hoogen H, Bor H. Seasonal incidence of stroke. Lancet. 1996; 347:1702-1703.

25. Reynolds E, Baron RB. Hypertension in women and the elderly. Postgrad Med. 1996; 100:58-69.

26. Biller J, Love BB. Diabetes and stroke. Med Clin North Am. 1993; 77:95-110. 
27. Stroke Prevention in Artrial Fibrillation Investigators. Adjusted-dose warfin versus low-intensity, fixed dose warfarin plus asprin for high-risk patients with atrial fibrillation. Lancet. 1996; 348:633-638.

28. Reunanen A, Takkunen H, Aroma A. Prevalence of intermittent claudication and its effect on mortality. Acta Med Scand. 1982; 211:249-256.

29. Criqui MH, Denenberg JO, Bird CE, Fronek A, Klauber MR, Langer RD. The correlation between symptoms and noninvasive test results in patients referred for peripheral arterial disease testing. Vascular Medicine. 1996; 1:65-71.

30. Burke GL, Evans GW, Riley WA, Sharrett AR, Howard G, Barnes RW, Rosamond W, Crow RS, Rautaharju PM, Heiss G. Arterial wall thickness is associated with prevalent cardiovascular disease in middle-aged adults. The Atherosclerosis Risk in Communities (ARIC) Study. Stroke. 1995; 26:386-391.
31. Criqui MH, Langer RD, Fronek A, Feigelson HS, Klauber MR, McCann TJ, Browner D. Mortality over a period of 10 years in patients with peripheral arterial disease. $\mathrm{N}$ Engl $\mathrm{J}$ Med. 1992; 326:381-386.

32. Buchwald H, Varco RL, Matts JP, Long JM, Fitch LL, Campbell GS, Pearce MB, Yellin AE, Edmiston WA, Smink $\mathrm{RD} \mathrm{Jr}$, et al. Effect of partial ileal bypass surgery on mortality and morbidity from coronary heart disease in patients with hypercholesterolemia. Report of the Program on the Surgical Control of the Hyperlipidemias (POSCH). N Engl J Med. 1990; 323:946-955.

33. Edwards JW, Manson JE, Hennekens CH, Buring JE. The primary prevention of coronary heart disease in women. $\mathrm{N}$ Engl J Med. 1995; 332:1758-1766.

34. Tamam N Mohamad, Yasmine Subhi Ali, Primary and Secondary Prevention of Coronary Artery Disease, WebMed, 2012 